\title{
The Performance of Cooperative Distributed Space Time Block Codes (CDSTBC) in Wireless System
}

\author{
Jacqueline. J. George,Mayada Abdelgadir, Mohamed Yousif and Ismail Hussein \\ College of Engineering \\ Sudan University of Science and Technology \\ Khartoum, Sudan \\ \{jaco_john, midoo-33, firewood60, isma3eel_z4\}@hotmail.com
}

\begin{abstract}
Mobile communication companies at present are looking for a $4 \mathrm{G}$ technology that aims at better reliability. This aim can be achieved by using Multiple Inputs Multiple Outputs (MIMO) system which is difficult to implement for the mobile terminals due to the limitations of the complexity, size and cost. So, these companies have been looking for a system which emulates a MIMO system. The cooperative relaying system has been proposed as a solution to be used to take the advantages of MIMO. The cooperative relaying system enables single antenna mobiles in a multi user environment to share their antennas and generate a virtual multiple antenna transmitters. In this paper, we investigate a single relay system that aims for a reliable communication. The main goal of this paper is to study the feasibility of the proposed single relay cooperative transmission in the mobile environment, and evaluate it with respect to the direct link transmission. Both systems are presented and a comparison in terms of bit error rate (BER) reveals that the single relay system performs better. The proposed single relay system needs an efficient coding technique to improve the performance in terms of reliability. Therefore, STBC as a coding technique is used, and the results proved that this technique decreases the BER.
\end{abstract}

Keywords- Cooperative relaying, amplify and forward, unit equal ratio combining, Alamouti scheme, cooperative distributed space time block codes.

\section{INTRODUCTION}

Multiple Inputs Multiple Outputs (MIMO) is a promising technology for future wireless communication systems [10]. By exploiting the multi-dimensional wireless channel created by multiple transmit and receive antennas, MIMO systems significantly increase the channel capacity and link robustness of wireless communication [6]. However, in some scenarios the wireless terminals may not be able to support multiple transmit antennas due to hardware limitations. In this case, it is possible to enable single antenna mobiles in a multi-user environment to share their antennas and generate a virtual multiple antenna transmitter that allows them to achieve transmit diversity [11]. This is known as cooperative relaying. The relay virtually plays a role of another transmitter and helps the source to communicate with the destination [7].

Space Time Block Coding (STBC) has recently emerged as a popular multi antenna communication scheme, owing to its simple code construction and maximal diversity gain.
Therefore the relay system using STBC as a coding technique has a significant impact on the overall performance of a wireless network [3].

The cooperative relay communication concept will play an important role in the cost effective provision of very high data rates and reliable communication in an almost-ubiquitous manner. This technology is fully in line with the designing rule of the Fourth Generation (4G) because its features are a crucial point for the success of the $4 \mathrm{G}$ cellular networks [15].

In this paper, the cooperative relaying system is introduced. Also, the STBC as a coding technique is used in this system which is called a Cooperative Distributed Space Time Block Codes (CDSTBC) System. The main objective of this paper is to test the performance of the single relay communications system compared with the direct transmission system in terms of bit error rate (BER) and to apply an efficient coding technique to this system to improve its performance.

\section{COOPERATIVE COMMUNICATION SYSTEM}

A Cooperative communications system uses several terminals which are called relays situated between the source (terminal that transmits information) and the destination (terminal that receives information) to improve their wireless communication [13]. The proposed relay channel model is composed of three terminals; a source that transmits information, a destination that receives information and a relay that both receives and transmits information as shown in Fig. 1.

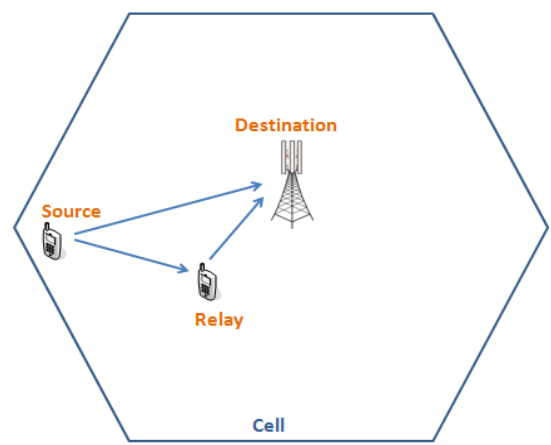

Figure 1. Three Cooperative Nodes Model 
In this system the relay uses the Amplify and Forward protocol (AAF) in its station. It down converts the received signal, amplifies it and up converts it to another frequency band prior to re-transmission [5]. At the destination, the two received signals are added using the Equal Gain Combining (EGC) method where all the received signals are summed coherently [14].

\section{THE CODING TECHNIQUE FOR THE SINGLE RELAY COOPERATIVE SYSTEM}

Space Time Codes (STCs) refer to the set of coding schemes that allow for the adjusting and optimization of joint encoding across multiple transmit antennas in order to maximize the reliability of a wireless link [4]. STC schemes exploit spatial diversity in order to provide coding and diversity gains over an uncoded wireless link. STC for the first time was proposed by Alamouti in 1997. This technique was called STBC. It is a popular technique amongst researchers of wireless communication [3].

The equivalent space time block coding transmission matrix for a two transmit, one receive antennas scheme with Rayleigh fading channel is given by

$$
x_{\text {Alamout }}=\left[\begin{array}{cc}
x_{1} & x_{2} \\
-x_{2}^{4} & x_{1}^{4}
\end{array}\right]
$$

Where:

$$
\begin{aligned}
& x_{2} \text { : is the transmitted symbol from the first transmit } \\
& \text { antenna at the time slot } t \text {. } \\
& \mathrm{n}_{\mathrm{z}} \text { : is the transmitted symbol from the second } \\
& \text { transmit antenna at time slot } \mathrm{t} \text {. } \\
& -\mathrm{x}_{\mathrm{z}}^{*} \text { : is the transmitted complex conjugate of } \mathrm{x}_{\mathrm{z}} \text { from } \\
& \text { the first transmit antenna at the time slot } \mathrm{t}+1 \text {. } \\
& \mathrm{x}_{2}^{-} \text {: is the transmitted complex conjugate of } \mathrm{x}_{2} \text { from } \\
& \text { the second transmit antenna at time slot } \mathrm{t}+1 \text {. }
\end{aligned}
$$

Let $x_{1}$ and $x_{i}$ assumed to be zero mean with unity variance. It is clear that the encoding is done in both space and time domain. As shown in table I, stream A from the first antenna contains $x_{2}$ and $-x_{z}^{*}$ and stream B from the second antenna contains $x_{\mathrm{s}}$ and $\mathrm{x}_{\mathrm{i}}^{*}$

\begin{tabular}{|c|c|c|}
\multicolumn{2}{c}{ TABLE I. } & Alamouti STBC Encoding \\
\hline Time Slot & $\begin{array}{c}\text { Antenna 1 } \\
\text { 'Stream A' }\end{array}$ & $\begin{array}{c}\text { Antenna 2 } \\
\text { 'Stream B' }\end{array}$ \\
\hline $\mathrm{t}$ & $\mathrm{x}_{1}$ & $\mathrm{x}_{\mathbf{z}}$ \\
\hline $\mathrm{t}+1$ & $-\mathrm{x}_{\mathrm{F}}^{*}$ & $\mathrm{x}_{1}^{*}$ \\
\hline
\end{tabular}

To emulate space time code in cooperative relay system, the Cooperative Distributed Space Time Coding (CDSTC) technique was used where the relay nodes are allowed to simultaneously transmit over the same channel. The term distributed comes from the fact that the virtual multi-antenna transmitter is distributed between randomly located relay nodes [16].

\section{A. System Model}

A simplified system model for the single cooperative relay distributed space time Block code is depicted in Fig. 2. This figure illustrates the uplink scenario where the system consists of three nodes, the source node located on the outer cell (the new cell after increasing the coverage) and both the relay and the Base station (destination) nodes are in the inner cell (the basic cell before increasing the coverage). The cooperative link operates in two time slots per packet [9].

In the first slot as shown in Fig. 2 (a), the source node transmits the first half of the Alamouti encoded sequence which is stream A to the relay. This signal matches that which would be sent from one antenna in a true two antenna Alamouti transmission as illustrates in table I. The relay node receives this signal and stores the raw samples in a buffer. In the second time slot as shown in Fig. 2 (b), the source node transmits the other half of the Alamouti encoded sequence which is stream B to the destination, and the relay transmits its stored copy of the first transmission. The destination node receives the superposition of these simultaneous transmissions. From the perspective of the destination, it receives a standard Alamouti encoded packet, where each of the spatial components was exposed to an independent channel.

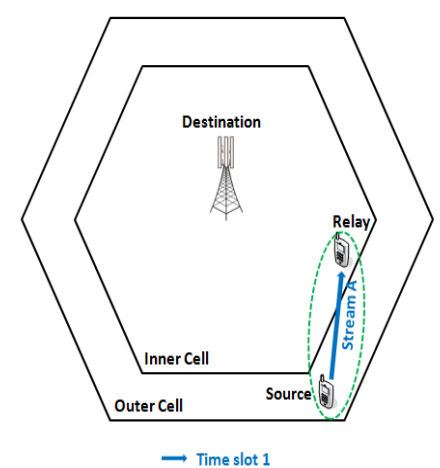

(a)

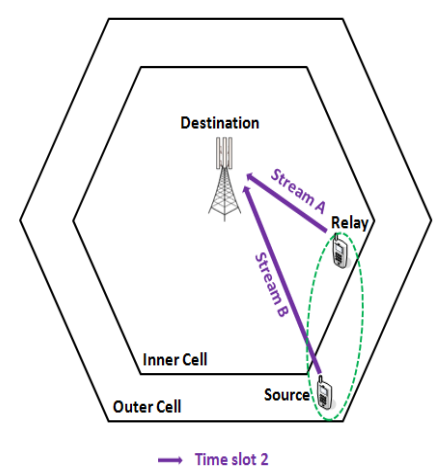

(b)
Figure 2. Single Cooperative Relay Distributed Space Time Block Code Scheme (a): The First Time Slot of the Transmission. (b): The Second Time Slot of the Transmission

\section{SIMULATION ANALYSIS OF THE COOPERATIVE DISTRIBUTED SPACE TIME BLOCK CODES (CDSTBC) SYSTEM}

Here a simulation of the performance of the system that uses a relay compared with the direct transmission in terms of the BER is introduced.Also STBC as a coding technique is applied in the single relay system to improve the reliability of this system. In this simulation the uplink scenario was considered; it assume that the relay uses Amplify and Forward (AAF) protocol to forward the data from the sender (mobile) to the destination (BS). The sender used BPSK modulation technique to modulate its data while the destination used the Equal Gain Combining method as a combining method. In Fig. 3 the BER versus the SNR is plotted to show the performance of the single relay cooperative Distributed Space Time Block Codes (CDSTBC) transmission. 


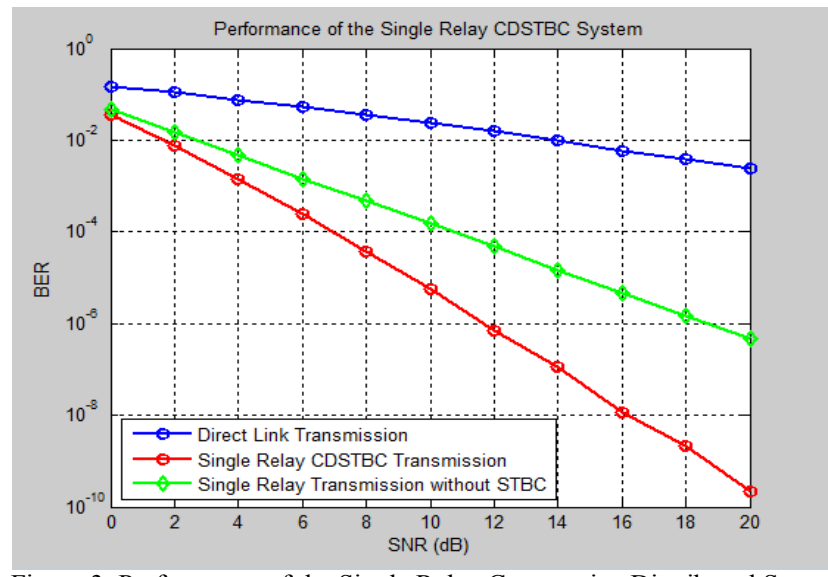

Figure 3. Performance of the Single Relay Cooperative Distributed Space Time Block Codes (CDSTBC) System

As shown in the graph Fig. 3, as the SNR increases, the BER of the three systems decreases. However, the result shows the bad performance of the direct link transmission compared with the other two systems. Also, to achieve a BER of about $10^{-6}$ the required SNR for the single relay with STBC is about $6 \mathrm{~dB}$ less than that of the single relay without STBC. Therefore, the single relay cooperative Distributed Space Time Block Codes (CDSTBC) system results in a better performance than the one without this technique.

Accordingly to reduce the BER, the above result indicates a significant increase in the coverage area for the CDSTBC over the direct transmission scheme under fairly the same bandwidth efficiency and fixed average transmitted power. The BS's coverage area can be expanded to cover the other mobiles in the other neighbour cells with minimum data loss. Therefore, the number of new cells is reduced.

\section{CONCLUSIONS}

The objective of this work was to give an overview of the cooperative relaying system. Also, STBC as a coding technique to improve the performance of this system in terms of reliability was analysed. The main goal of this paper was to show the possible benefits of a wireless transmission using cooperative diversity to increase the performance by using Matlab environment. The diversity is realized by building a system using a third station as a relay. The data is sent directly from the base to the mobile or via the single relay station. Such a system has been simulated to see the performance of the AAF protocol and EGC combining method. The results show that the single relay system performs better than the direct link transmission in terms of BER. The results also showed that using the STBC as a coding technique in a single relay system decreases the BER and therefore increase the coverage area.

\section{REFERENCES}

[1] Frank Fitzek, Marcos D. Katz, "Cooperation in Wireless Networks: Principles and Applications", Springer, 2008.

[2] A. B. Gershman, N.D. Sidiropoulos,"Space-Time Processing for MIMO Communications", Wiley, 2005.

[3] Hamid Jafarkhani ,"Space-Time Coding Theory and Practice", Campridge, 2005

[4] Mohinder Jankiraman, "Space-Time Codes and MIMO Systems", Artech House, 2004.

[5] K.J. Ray Liu, Ahmed K. Sadek, Weifeng Su, "Cooperative Communications and Networking", Campridge, 2009.

[6] Volker Kuhn, "Wireless Communications over MIMO Channels Applications to CDMA and Multiple Antenna Systems", John Wiley \& Sons Ltd, 2006.

[7] Patrick Murphy, Ashutosh Sabharwal and Behnaam Aazhang, "On Building a Cooperative Communication System", Testbed Implementation and First Results, Rice University, 2007.

[8] Peter Rost, "BER Performance Evaluation of a Continuous Transmission Cooperative Relaying Protocol", Dresden, Germany,2005.

[9] Klaus Witrisal, "Narrowband Systems - Principle of Diversity and MIMO Systems", Technical University Graz, Austria, 2007.

[10] Shi Min, "An Introduction to MIMO Technology", Ohtsuki Lab. 2007.

[11] Kun Pang, "Collaborative HARQ Schemes for Cooperative Diversity Communications in Wireless Networks", University of Sydney, February 2008.

[12] Lei Xiao, "Cooperative Relaying Strategies for Wireless Communication Systems", University of Notre Dame, December 2008.

[13] Y.-W. Peter Hong, Wan-Jen Huang and C.-C. Jay Kuo, "Cooperative Communications and Networking, Tchnologies and system design", Springer, 2010.

[14] Ernesto Zimmermann, Patrick Herhold, Gerhard Fettweis, "On the Performance of Cooperative Relaying Protocols in Wireless Networks", D-01062 university Dresden, Germany,2006. 